\title{
Fractal Shaped Antenna based tri-band Energy Harvester
}

\author{
Srijan Datta ${ }^{1}$, Kaustav Kar ${ }^{1}$, Manimala Pal $^{2}$ and Rowdra Ghatak ${ }^{1}$ \\ ${ }^{1}$ ECE Dept., Microwave and Antenna Research Laboratory, National Institute of Technology Durgapur \\ ${ }^{2}$ Radio Engineering and Antenna Laboratory, ECE Dept., NSHM Knowledge Campus, Durgapur-Group of \\ Institutions, Durgapur, West Bengal, INDIA \\ Email: srijan.rio@gmail.com
}

\begin{abstract}
Energy can be conserved by reusing what has already been spent. Such type of energy is readily available in the electromagnetic form (ambient RF energy). Signals broadcasted from AM, FM, cellular base stations and millions of other wireless devices can be converted to DC power. However, the main roadblock in this field of research is the level of power that ambient radiation carries. High efficiency antennas and rectifier circuits are required to harvest a fair amount of energy that can be used by low power devices. This paper presents the design of a novel multiband fractal antenna and a rectifier circuit that can be used to harvest ambient RF energy.
\end{abstract}

\section{Introduction}

Large scale use of alternate sources of energy has become a very important field of research as the conventional sources of energy are getting depleted. The choice of such an alternate source mainly depends on its availability. One such form of energy is the ambient RF energy which is present almost everywhere. The electromagnetic radiation of millions of wireless devices, cellular base stations, AM and FM radio base stations can be used to charge low power devices like RFID and wireless sensor networks, thereby partially or completely removing the concept of batteries. However, the amount of RF energy that can be harvested depends on the following important factors- the power emitted from the source, the gain of the RF source antenna, sensitivity and reflection coefficient characteristics of the receiving antenna and the RF-DC conversion efficiency of the rectifier circuit. Fig 1 shows the schematic of a RF harvester.

The fractal Koch first appeared in 1904 [1]. Fractal geometry uses self-similar design to maximize the length or inner perimeter. Therefore, it is quite effective in designing compact multiband antennas for energy harvesting. V. Hebelka et. al.[2] implemented a modified design of the Koch geometry to design a dual band antenna for energy harvesting applications. Few works are reported in literature consisting of fractal antennas as the receiving antenna of rectenna. [3] studies a fractal antenna design for multiband harvesting. The area of the antenna significantly increases while including the $900 \mathrm{GSM}$ band.

Rectifier circuits with high RF to DC power conversion efficiency are required to extract a fair amount of power from the frequency bands. E. Khansalee et. al. [4] designed a rectifier circuit with a single impedance matching network for harvesting energy from two bands. The circuit had a peak efficiency of $40 \%$ at $10 \mathrm{dBm}$ input power. Z. Popovic et. al. [5] introduced a RF energy harvesting module which harvests energy from incident plane waves which have power densities that vary over time and space. They used distributed antenna arrays and a reconfigurable DC combining network to efficiently harvest energy over a wide range of frequencies.

Fractal shapes can be implemented to produce wide-band as well as multi-band antennas. However, rectifier circuit design, specifically a single impedance matching network design for wideband antennas is quite difficult [6]. Hence a multi-band antenna is preferred for selective energy harvesting from the resonating frequency bands.

This paper proposes the design of a hybrid fractal antenna which implements a combination of Sierpinski carpet and a modified Koch curve. The target frequency bands are the GSM 900, 1800 and the WLAN $2.4 \mathrm{GHz}$. The design of an efficient rectifier circuit has also been proposed to harvest energy from the three bands. In the next section, the design and results of the hybrid fractal antenna are reported followed by the third section which presents the design of the multiband rectifier circuit.

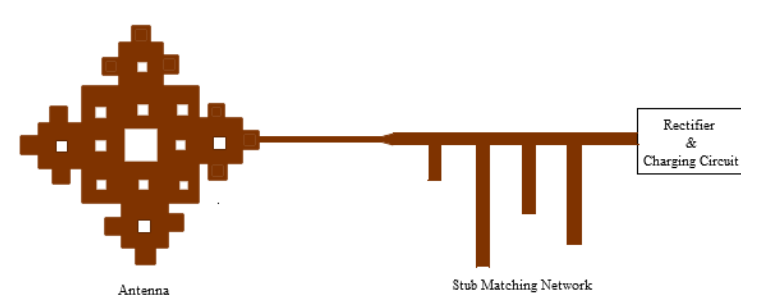

Figure 1: RF energy harvesting module.

\section{Hybrid Fractal Antenna}

The antenna is based on the concept of hybrid fractal antenna [7]. It implements a combination of the classic Sierpinski carpet and Koch curve geometries. Both the fractal geometries are implemented to their second iteration. Introduction of the Koch curve to the Sierpinski carpet helps in reduction of the patch size of the normal Sierpinski carpet antenna [8]. It also gives rise to better return loss characteristics [9]. Moreover, the Koch curve at the feeding 
Table 1

\begin{tabular}{|c|c|c|c|c|c|}
\hline Parameter & Lp & Lg & Wg & Wf & Fg \\
\hline Dimension(mm) & 30 & 79 & 66 & 1 & 1 \\
\hline
\end{tabular}

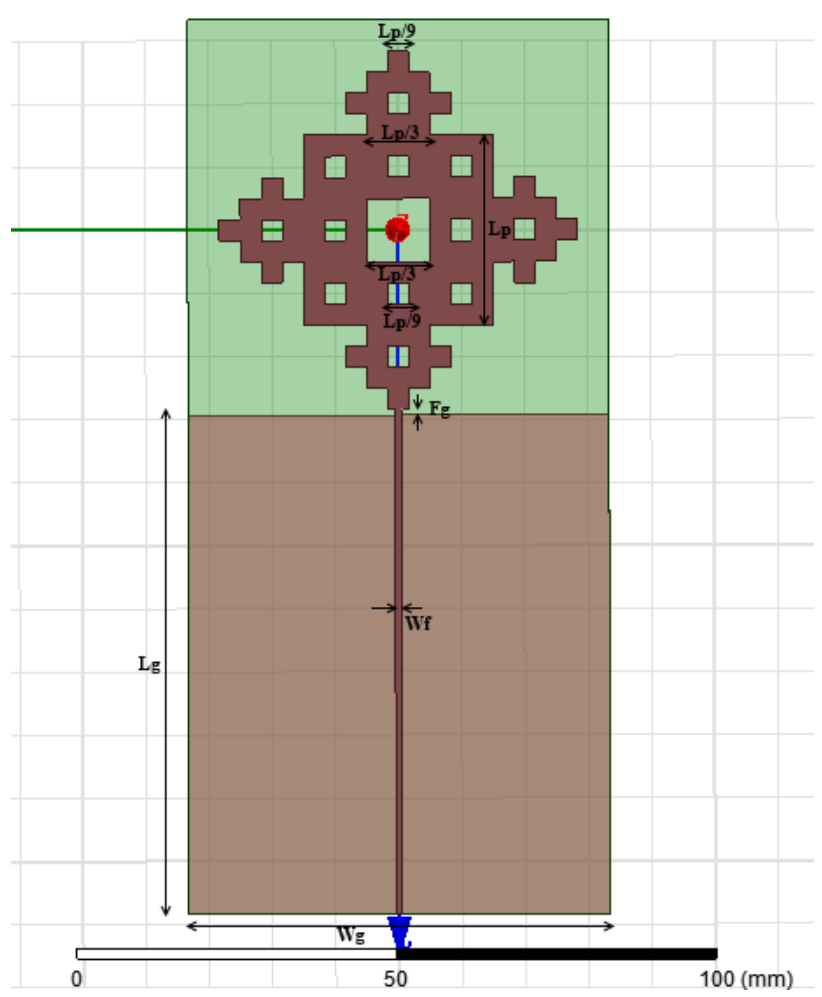

Figure 2: Proposed hybrid fractal shaped antenna

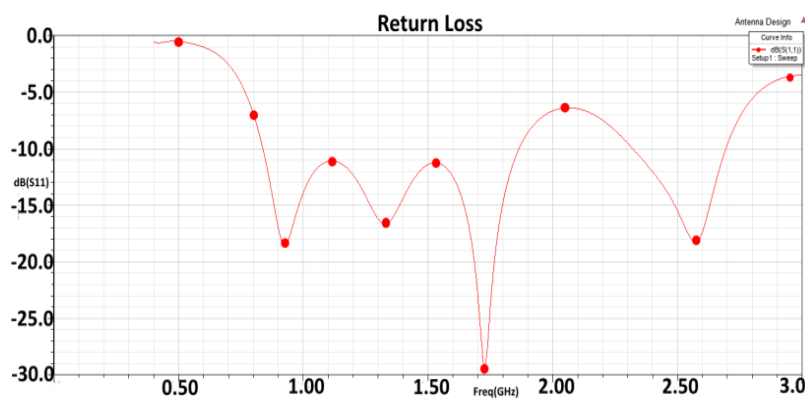

Figure 3: $\mathrm{S}_{11}$ of antenna.

edge provides a stepped feed to the antenna which improves the bandwidth characteristics [10].

Fig 2 shows the proposed antenna design. Table 1 contains the dimensions of the design parameters. The antenna is built on $1.6 \mathrm{~mm}$ FR4 substrate with a microstrip feed line of width $1 \mathrm{~mm}(\mathrm{Wf})$ which encompasses a total area of $14 \times 6.6$ $\mathrm{cm}^{2}$. Partial ground plane $(\mathrm{Lg} \times \mathrm{Wg})$ with feed gap $(\mathrm{Fg})$ optimisation technique is used to obtain multiband impedance matching in the desired frequencies [11][12].

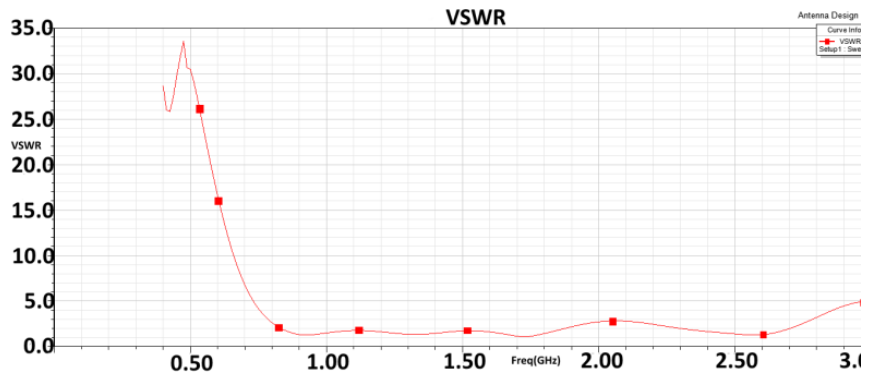

Figure 4: VSWR plot of antenna

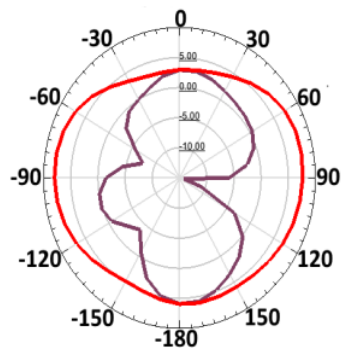

Fig. 5a

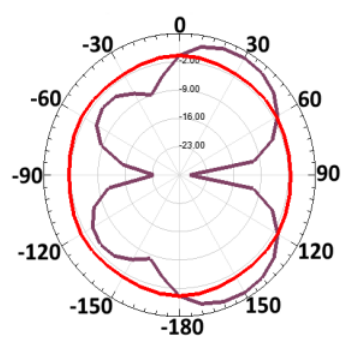

Fig. $5 b$
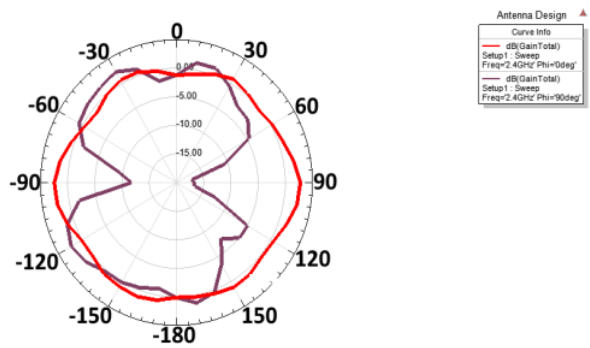

Fig. 5c

Figure 5: Radiation pattern in E and $\mathrm{H}$ plane (a) $900 \mathrm{MHz}$ (b) $1.8 \mathrm{GHz}$ (c) $2.4 \mathrm{GHz}$

A parametric study was done to find out the best possible feed gap length and the resulting $S_{11}$ variation for different feed gaps has been shown in figure 6 . From the figure it can be seen that for gap length of $0 \mathrm{~mm}$ the $\mathrm{S}_{11}$ characteristics is shifted to the right at 0.9 and $2.4 \mathrm{GHz}$. When the gap length is increased to $2 \mathrm{~mm}$, the $\mathrm{S}_{11}$ value improves at $1.8 \mathrm{GHz}$. However the value goes below $-10 \mathrm{~dB}$ at $2.4 \mathrm{GHz}$. Thus an optimum gap length of $1 \mathrm{~mm}$ was used to design the antenna. 


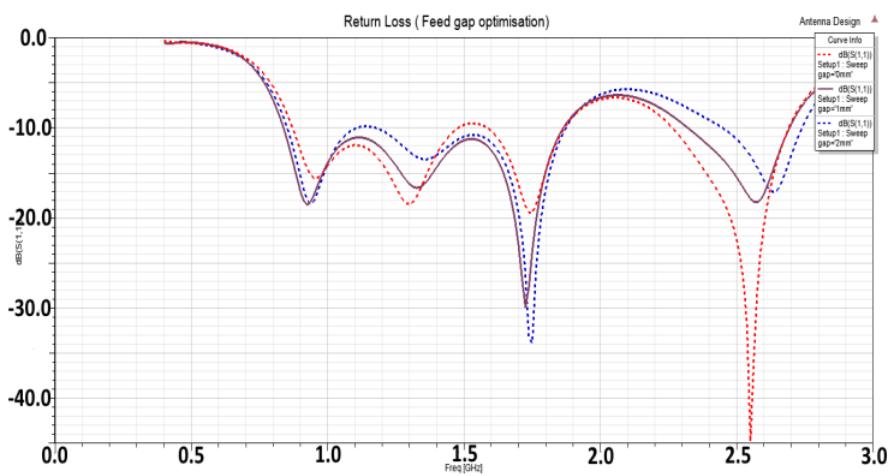

Figure 6: $\mathrm{S}_{11}$ of antenna with feed gap (a) $0 \mathrm{~mm}(\mathrm{red})$ (b) $1 \mathrm{~mm}$ (grey) (c) $2 \mathrm{~mm}$ (blue)

Return loss characteristics of the antenna are reported in Fig. 3. The values of $S_{11}$ at working frequencies are $-19 \mathrm{~dB}$ at 0.9 $\mathrm{GHz},-15 \mathrm{~dB}$ at $1.8 \mathrm{GHz}$ and $-12 \mathrm{~dB}$ at $2.4 \mathrm{GHz}$. The VSWR plot (Fig. 4) is also less than 2 for the required bands. Fig. 5 $\mathrm{a}, \mathrm{b}$ and $\mathrm{c}$ show the radiation pattern in $\mathrm{E}$ and $\mathrm{H}$ plane at the centre frequency of the working bands.

The $\mathrm{S}_{11}$ plot shows two widebands which allows us to design a rectifier circuit according to our needs, i.e., either we can select individual frequencies and harvest energy from them or we can extract energy from a wide range of frequencies. In the next section we have designed a rectifier that implements multi-band impedance matching to harvest energy from individual frequencies.

\section{Multi-band Rectifier Circuit Design}

The proposed rectifier circuit is based on the Greinacher voltage doubler topology [13]. The schematic of the rectifier circuit is given in Fig. 7. This topology is quite effective in extracting power from a RF source.

The diodes D1 and D2 used in the circuit are Agilent HSMS 2852 zero bias Schottky detector diodes. The diodes are in series and are connected with two capacitors C1 and C2 which have a value of $1 \mathrm{pf}$ each. The zero bias Schottky diode has a low forward voltage (Vf) from $125 \mathrm{mV}$ to 250 $\mathrm{mV}$, breakdown voltage $(\mathrm{Vb})$ of $3.8 \mathrm{~V}, 25 \mathrm{Ohms}$ of series

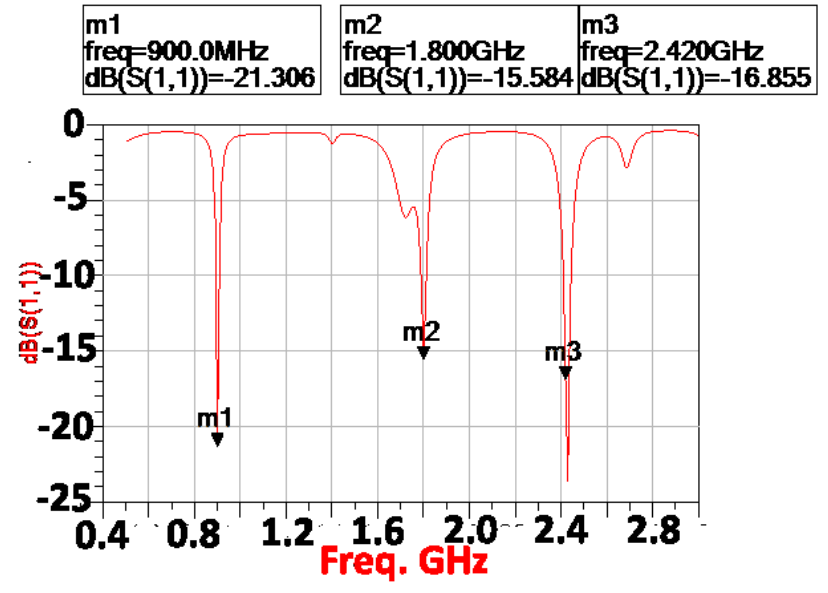

Figure 8: $\mathrm{S}_{11}$ variation of rectifier circuit.

resistance (Rs) and 0.18 pf of zero bias junction capacitance (Cj0).

The most vital part of the circuit is the matching network which matches the antenna impedance to the rectifier circuit impedance. Matching networks for multiband energy harvesters are of two types- single matching network [14] and multiple matching networks for individual resonating frequencies [6] which use power combining techniques. In this paper, we have designed a single matching network which has been optimised in ADS. The reflection coefficient characteristics of the rectifier circuit are given in Fig. 7. The values at target frequencies are $-21.306 \mathrm{~dB},-15.584 \mathrm{~dB}$ an $-16.855 \mathrm{~dB}$ which shows that the incoming signal in those bands would face minimum reflection.

The performance of the circuit is displayed in Fig. 9 and 10. The simulated results consist of efficiency and output voltage for a load of $1 \mathrm{kOhm}$. The harvesting circuit has a peak efficiency of $9 \%$ in the $900 \mathrm{MHz}$ band, $30.5 \%$ in 1.8 $\mathrm{GHz}$ band and $8.7 \%$ in $2.4 \mathrm{GHz}$ band. A sweep of the input power from $-40 \mathrm{dBm}$ to $20 \mathrm{dBm}$ gives a peak overall efficiency of $21.566 \%$ for an input power of $12.5 \mathrm{dBm}$. The maximum voltage generated from the circuit is 3.433 for an input power of $14.5 \mathrm{dBm}$ (refer to figure 10) when all the bands are supplying power simultaneously.

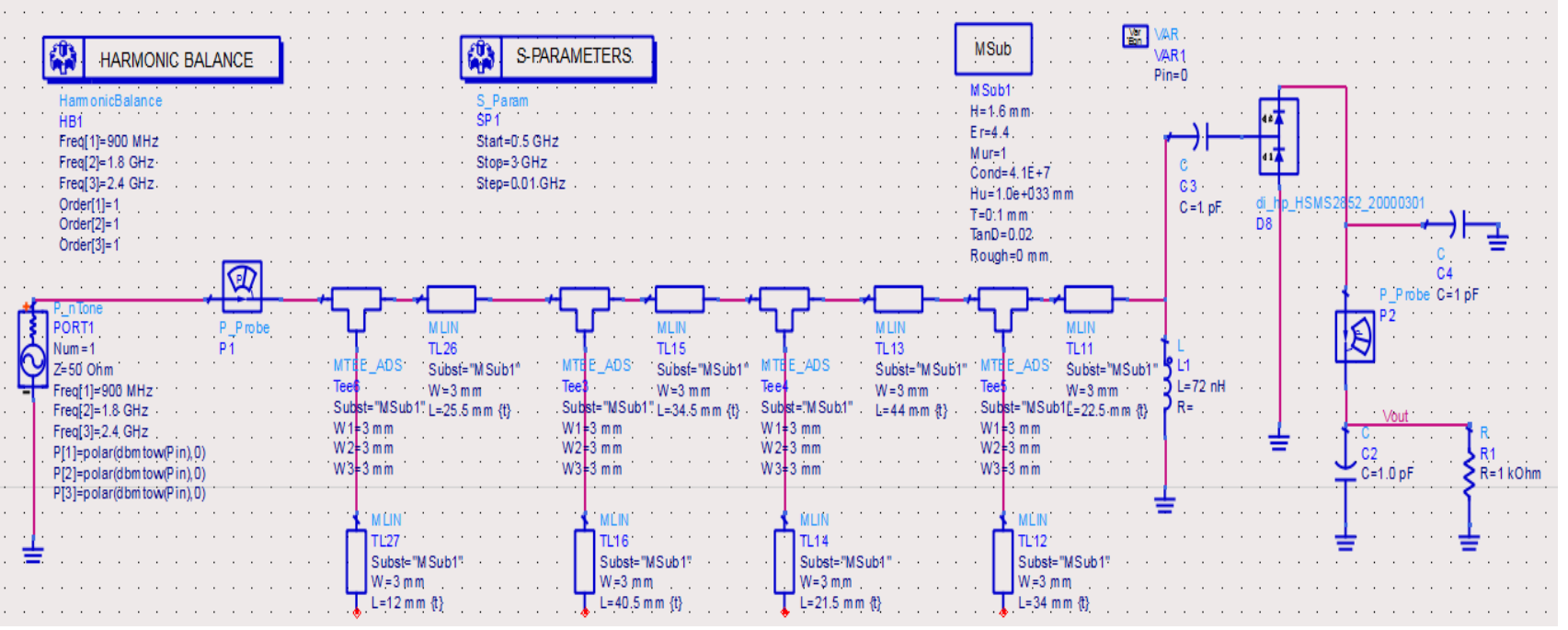

Figure 7: Rectifier circuit schematic with matching network. 


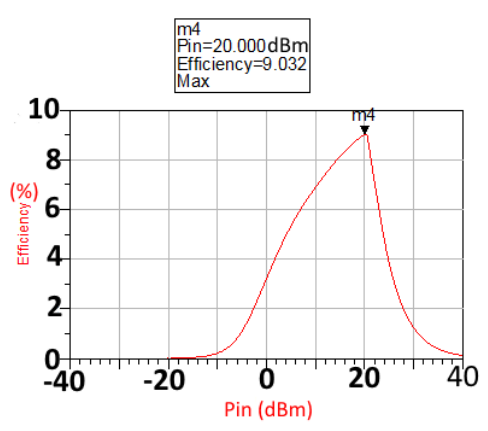

Figure 9a

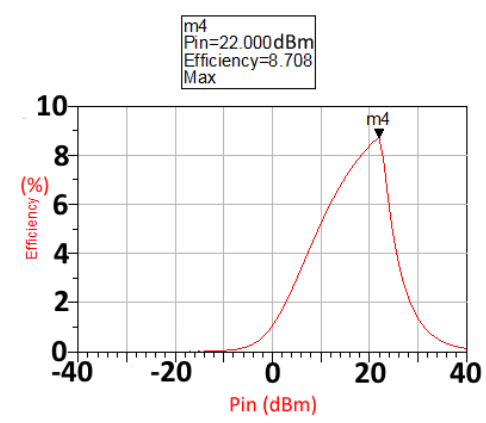

Figure 9c

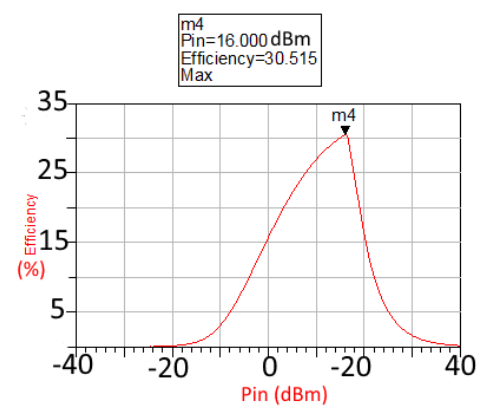

Figure $9 b$

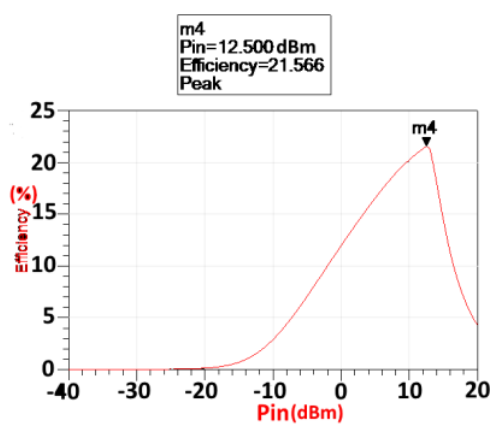

Figure 9d

Figure 9: Efficiency of rectifier circuit vs input power at (a) $900 \mathrm{MHz}$ (b) $1.8 \mathrm{GHz}$ (c) $2.4 \mathrm{GHz}$ (d) Overall

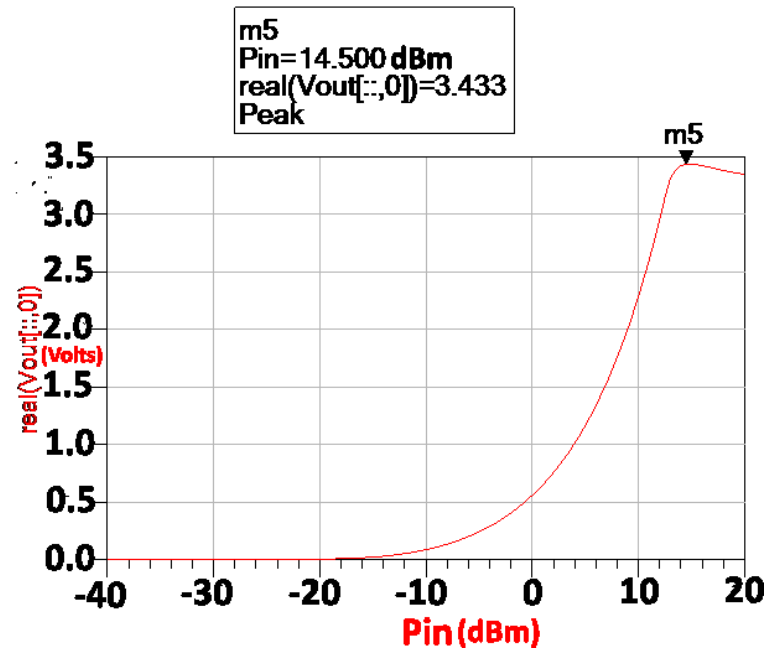

Figure 10: Output Voltage across load resistance of 1 $\mathrm{KOhm}$

\section{Conclusion}

Sustainable sources of energy have become a topic of foremost importance in our current times. This reason is likely to propel RF harvesting to become a forerunner in the field of technology. Moreover, Internet of Things greatly depends on the progress of RF harvesting along with wireless power transfer. This paper presents a novel hybrid fractal antenna design to capture ambient RF energy in GSM 900, 1800 and WLAN $2.4 \mathrm{GHz}$ bands. A multiband rectifier circuit to convert the incident RF power to DC power is reported with a peak efficiency of $21.57 \%$ and a maximum output voltage of $3.43 \mathrm{~V}$ is obtained. Future work includes implementation and integration of the antenna and rectifier circuit to build a compact multiband RF harvester.

\section{References}

[1] H. 0. Peitgen, H. Jurgens, and D. Saupe, Chaos and Fractals. New Frontiers of Science, New York, Springer-Verlag, Inc., 1992.

[2] V. Hebelka, J. Velim and Z. Raida, "Dual band Koch antenna for RF energy harvesting," 10th European Conference on Antennas and Propagation (EuCAP), pp. 1-3, 2016.

[3] Z. Zhou, W. Liao, Q. Zhang, F. Han and Y. Chen, "A multi-band fractal antenna for RF energy harvesting," 2016 IEEE International Symposium on Antennas and Propagation (APSURSI), Fajardo, 2016, pp. 617-618.

[4] E. Khansalee, Y. Zhao, E. Leelarasmee and K. Nuanyai, "A dual-band rectifier for RF energy harvesting systems," 11th International Conference on Electrical Engineering/Electronics,

Computer, Telecommunications and Information Technology (ECTI-CON), pp. 1-4, 2014. 
[5] Z. Popović et al., "Scalable RF Energy Harvesting," in IEEE Transactions on Microwave Theory and Techniques, vol. 62, no. 4, pp. 1046-1056, April 2014.

[6] D. Pavone, A. Buonanno, M. D'Urso, and F. Della Corte, "Design considerations for radio frequency energy harvesting devices," Progress In Electromagnetics Research B, Vol. 45, pp. 19-35, 2012.

[7] R. A. Kumar, Y. K. Choukiker and S. K. Behera, "Design of hybrid fractal antenna for UWB application," 2012 International Conference on Computing, Electronics and Electrical Technologies (ICCEET), Kumaracoil, 2012, pp. 691-693.

[8] M. K. A. Rahim, N. Abdullah and M. Z. A. Abdul Aziz, "Microstrip Sierpinski carpet antenna design," 2005 Asia-Pacific Conference on Applied Electromagnetics, 2005, pp. 4 pp.-.

[9] B. K. Jeemon, K. Shambavi and Z. C. Alex, "A multifractal antenna for WLAN and WiMAX application," 2013 IEEE Conference on Information \& Communication Technologies, JeJu Island, 2013, pp. 953-956.

[10]H. S. Mewara, M. Sharma, M. M. Sharma and A. Dadhich, "A novel ultra-wide band antenna design using notches and stairs," 2014 International Conference on Signal Propagation and Computer Technology (ICSPCT 2014), Ajmer, 2014, pp. 425-429.

[11] John, M., Ammann, M.J.: 'Optimization of impedance bandwidth for the printed rectangular monopole antenna', Microw. Opt. Tech. Lett., 2005, 47, (2), pp. $153-154$

[12] J. Kumar and S. S. Shirgan, "Compact Partial Ground Plane 1x2 Patch Antennas," 2014 International Conference on Computational Intelligence and Communication Networks, Bhopal, 2014, pp. 33-37.

[13]Z. Khonsari, T. Björninen, L. Sydänheimo, M. Tentzeris and L. Ukkonen, "Inkjet-printed monopole antenna and voltage doubler on cardboard for RF energy harvesting," IEEE International Symposium on Antennas and Propagation \& USNC/URSI National Radio Science Meeting, pp. 1312-1313, 2015.

[14]Binboga Siddik Yarman, Metin Sengul, P. Lindberg and A. Rydberg, "A single matching network design for a double band PIFA antenna via simplified real frequency technique," 2006 Asia-Pacific Microwave Conference, Yokohama, pp. 1325-1328, 2006. 